\title{
A LOFAR DETECTION OF THE LOW-MASS YOUNG STAR T TAU AT $149 \mathrm{MHz}$
}

\author{
Colm P. Coughlan ${ }^{1}$, Rachael E. Ainsworth ${ }^{1}$, Jochen Eislöffel ${ }^{2}$, Matthias Hoeft $^{2}$, Alexander Drabent $^{2}$, \\ Anna M. M. Scaife ${ }^{3}$, Tom P. Ray ${ }^{1}$, Martin E. Bell ${ }^{4,5}$, Jess W. Broderick ${ }^{6}$, Stéphane Corbel ${ }^{7,8}$, \\ Jean-Mathias Grießmeier $^{8,9}$, Alexander J. Van der Horst ${ }^{10}$, Joeri van Leeuwen ${ }^{6,11}$, Sera Markoff ${ }^{11}$, \\ Malgorzata Pietka ${ }^{12}$, Adam J. Stewart ${ }^{12}$, Ralph A. M. J. Wijers ${ }^{11}$, And Philippe Zarka ${ }^{8,13}$ \\ ${ }^{1}$ Dublin Institute for Advanced Studies, School of Cosmic Physics, 31 Fitzwilliam Place, Dublin D02 XF86, Ireland \\ 2 Thüringer Landessternwarte, Sternwarte 5, D-07778, Tautenburg, Germany \\ ${ }^{3}$ Jodrell Bank Centre for Astrophysics, School of Physics and Astronomy, The University of Manchester, Oxford Road, Manchester M13 9PL, UK \\ ${ }^{4}$ CSIRO Astronomy and Space Science, P.O. Box 76, Epping, NSW 1710, Australia \\ ${ }^{5}$ ARC Centre of Excellence for All-sky Astrophysics (CAASTRO), The University of Sydney, NSW 2006, Australia \\ ${ }^{6}$ ASTRON, the Netherlands Institute for Radio Astronomy, Postbus 2, 7990 AA Dwingeloo, The Netherlands \\ ${ }^{7}$ Laboratoire AIM (CEA/IRFU-CNRS/INSU-Université Paris Diderot), CEA DSM/IRFU/SAp, F-91191 Gif-sur-Yvette, France \\ ${ }^{8}$ Station de Radioastronomie de Nançay, Observatoire de Paris, PSL Research University, CNRS, Univ. Orléans, F-18330 Nançay, France \\ ${ }^{9}$ LPC2E-Université d'Orléans/CNRS, F-45071 Orléans cedex 2, France \\ ${ }^{10}$ Department of Physics, The George Washington University, 725 21st Street NW, Washington, DC 20052, USA \\ ${ }^{11}$ Anton Pannekoek Institute for Astronomy, University of Amsterdam, Postbus 94249, $1090 \mathrm{GE}$ Amsterdam, The Netherlands \\ 12 Astrophysics, Department of Physics, University of Oxford, Keble Road, Oxford OX1 3RH, UK \\ ${ }^{13}$ LESIA, Observatoire de Paris, CNRS, PSL/SU/UPMC/UPD/SPC, Place J. Janssen, F-92195 Meudon, France \\ Received 2016 August 8; revised 2016 November 2; accepted 2016 November 9; published 2017 January 13
}

\section{ABSTRACT}

Radio observations of young stellar objects (YSOs) enable the study of ionized plasma outflows from young protostars via their free-free radiation. Previous studies of the low-mass young system T Tau have used radio observations to model the spectrum and estimate important physical properties of the associated ionized plasma (local electron density, ionized gas content, and emission measure). However, without an indication of the low-frequency turnover in the free-free spectrum, these properties remain difficult to constrain. This paper presents the detection of T Tau at $149 \mathrm{MHz}$ with the Low Frequency Array (LOFAR) - the first time a YSO has been observed at such low frequencies. The recovered total flux indicates that the free-free spectrum may be turning over near $149 \mathrm{MHz}$. The spectral energy distribution is fitted and yields improved constraints on local electron density $\left((7.2 \pm 2.1) \times 10^{3} \mathrm{~cm}^{-3}\right)$, ionized gas mass $\left((1.0 \pm 1.8) \times 10^{-6} M_{\odot}\right)$, and emission measure $\left((1.67 \pm 0.14) \times 10^{5} \mathrm{pc} \mathrm{cm}^{-6}\right)$.

Key words: radiation mechanisms: thermal - radio continuum: stars - stars: individual (T Tauri) - stars: pre-main sequence - stars: protostars

\section{INTRODUCTION}

Classical T Tauri stars are pre-main-sequence stars that grow in mass through accretion from a surrounding circumstellar disk. They are observed to drive jets, which are believed to remove excess angular momentum from the circumstellar disk, thereby allowing accretion to proceed. These young stellar objects (YSOs) are typically detected at centimeter or longer wavelengths via free-free emission from their collimated, ionized outflows (see, e.g., Anglada et al. 2015). The observed free-free spectrum is characterized by a flat or positive powerlaw spectral index $\alpha$ (where the flux density $S_{\nu} \propto \nu^{\alpha}$ at frequency $\nu$ ) with a value of -0.1 for optically thin emission, +2 for optically thick emission, and intermediate values for partially opaque plasma (see, e.g., Scaife 2013).

Historically, with typical flux densities at centimeter wavelengths of $\lesssim 1 \mathrm{mJy}$ and a tendency to weaken at lower frequencies, observations of YSOs have been confined to frequencies $>1 \mathrm{GHz}$ (wavelengths $\lambda<30 \mathrm{~cm}$ ). However, with their free-free spectra expected to turn over at low frequencies as the emitting medium transitions from optically thin to thick (Scaife 2013), the study of YSOs at low frequencies offers a promising window into the physical parameters of their jets. Recent work by Ainsworth et al. $(2014,2016)$ has used the Giant Metrewave Radio Telescope (GMRT) to begin studying YSOs at very low radio frequencies $(323-608 \mathrm{MHz})$ and shows the feasibility of observing YSOs with interferometers such as the Low Frequency Array (LOFAR) and, ultimately, the Square Kilometre Array.

Ainsworth et al. (2016) discussed the importance of investigating the radio emission from young stars at low radio frequencies. Physical properties of the ionized plasma, such as the electron density, ionized gas mass, and emission measure, can be well constrained once the low-frequency turnover (between optically thin and thick behavior) in the free-free spectrum has been identified. These GMRT results demonstrated that the low-frequency turnover occurs at frequencies lower than $323 \mathrm{MHz}$ for their small target sample. Specifically, these authors showed that the prototype of an entire class of low-mass YSOs (Joy 1945), T Tau, would be well suited for further investigation with LOFAR because they estimate the turnover frequency to occur around $\sim 200 \mathrm{MHz}$. They identified the sensitivity and resolution of LOFAR at $150 \mathrm{MHz}$ as being well suited to constraining the size of the free-free emitting region in $\mathrm{T}$ Tau.

Low-frequency observations of young stars are also important for another reason: searching for non-thermal (synchrotron) emission from YSO jets (see, e.g., CarrascoGonzález et al. 2010). The existence of such emission was originally considered surprising, since the velocities of YSO jets are much lower than those of active galactic nuclei; however, recent modeling (e.g., Padovani et al. 2016) has 
shown how YSO jets can accelerate particles to relativistic energies through diffusive shock acceleration. Moreover, the successful detection of polarized emission associated with nonthermal processes is now considered an important window into the magnetic field environment of the jet. It is also notable that a large fraction of YSOs detected with the Very Large Array (VLA) in the Gould Belt Survey of Dzib et al. (2015) are consistent with non-thermal coronal emission. Flux densities from non-thermal emission processes increase with decreasing frequency, which should make them easier to detect with instruments such as LOFAR and the GMRT.

T Tau (J2000: $042159.4+1932$ 06.4) is a well-studied triple system located in the Taurus-Auriga Molecular Cloud at a distance of $148 \mathrm{pc}$ (Loinard et al. 2007b). The triplet consists of an optically visible star, T Tau N, and an optically obscured binary $0.77(\approx 100 \mathrm{au})$ to the south, T Tau Sa and Sb, visible at infrared and longer wavelengths and with a projected separation of 0." $13(\approx 19 \mathrm{au}$; Dyck et al. 1982; Koresko 2000; Köhler et al. 2008). Two large-scale outflows have been observed from the system (Reipurth et al. 1997; Gustafsson et al. 2010): an east-west outflow from $\mathrm{T}$ Tau $\mathrm{N}$ that terminates at the Herbig-Haro object $\mathrm{HH} 155$ (position angle $\sim 65^{\circ}$ with inclination angle to the plane of the sky $i \sim 80^{\circ}$ ) and a southeast-northwest flow associated with HH 255 and HH 355 that is thought to emanate from $\mathrm{T}$ Tau Sa (position angle $\sim 345^{\circ}$ with $i \leqslant 10^{\circ}$ ). A separate molecular flow in the southwest direction is associated with $\mathrm{T}$ Tau Sb (Gustafsson et al. 2010). $\mathrm{T}$ Tau $\mathrm{N}$ appears to be surrounded by an accretion disk that is nearly face-on, $\mathrm{T}$ Tau $\mathrm{Sa}$ is surrounded by a disk that is nearly edge-on, and both $\mathrm{T}$ Tau $\mathrm{Sa}$ and $\mathrm{T} \mathrm{Tau} \mathrm{Sb}$ are apparently surrounded by an edge-on circumbinary torus, which is responsible for the large extinction toward T Tau S (see, e.g., Loinard et al. 2007a, for an overview of the T Tau system).

At radio (centimeter) wavelengths, previous observations with high angular resolution made with the VLA resolve the northern and southern components of the T Tau triple system but do not resolve the southern binary (Schwartz et al. 1986; Skinner \& Brown 1994; Johnston et al. 2003; Smith et al. 2003; Loinard et al. 2007a). Figure 1 presents the multi-epoch observations at 5, 8, and $15 \mathrm{GHz}$ of Johnston et al. (2003). The emission from $\mathrm{T}$ Tau $\mathrm{N}$ does not exhibit a large degree of time variability and has a spectral index $\alpha \simeq 1$, indicating that the emission is thermal in origin (e.g., from a dense ionized stellar wind, see also Loinard et al. 2007a). In contrast, the emission from $\mathrm{T}$ Tau $\mathrm{S}$ shows a high degree of time variability over $\simeq 20$ yr and usually has a flat spectral index $(\alpha \simeq 0)$, which is normally indicative of optically thin free-free radiation. However, both $\mathrm{T}$ Tau $\mathrm{N}$ and $\mathrm{S}$ radio sources exhibit circular polarization, indicating that the emission from each component has contributions from gyrosynchrotron radiation due to magnetic activity (Skinner \& Brown 1994; Ray et al. 1997; Johnston et al. 2003).

At all epochs, the southern radio source is heavily dominant (with flux density ratio of order 6:1 between the southern and northern radio components, see Figure 1) and therefore makes the major contribution to the spectrum (and the variability) of the unresolved observations (lower-resolution observations suggest a ratio 10:1, see, e.g., Scaife 2011). Johnston et al. (2003) suggest that the southern radio source itself is dominated by the optically less luminous member, T Tau Sb. This is further supported by observations at very high angular

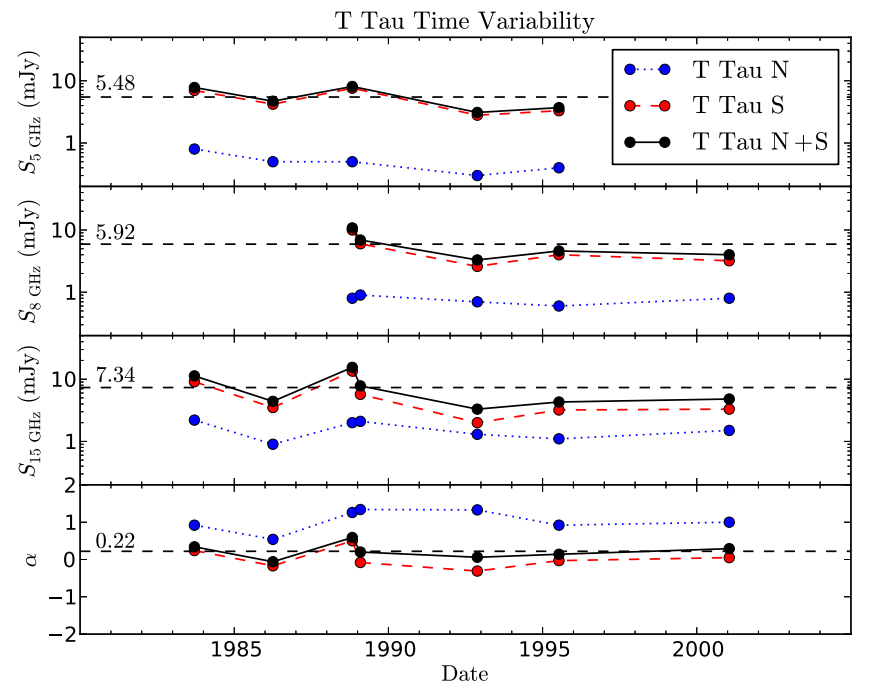

Figure 1. Time dependence of the flux density (at 5, 8, and $15 \mathrm{GHz}$ ) and spectral index ( $\alpha$, calculated between 5 and $15 \mathrm{GHz}$ except for epochs 1989 and 2001, which were calculated between 8 and $15 \mathrm{GHz}$ ) of the T Tau system from Johnston et al. (2003, data are from their Table 2). The (blue) dotted lines show the measurements for $\mathrm{T}$ Tau $\mathrm{N}$, the (red) dashed lines show the measurements for T Tau S and the (black) solid lines shows the combined, total measurement of the (T Tau $\mathrm{N}+\mathrm{S})$ system. The mean values for the flux densities and spectral index of the total $\mathrm{T}$ Tau $\mathrm{N}+\mathrm{S}$ system are quoted on the left of each plot and shown as horizontal (black) dashed lines. It is clear that the total flux density and spectral index are dominated by the variable emission from $\mathrm{T}$ Tau $\mathrm{S}$ (which is more extreme at higher frequencies) and that any unresolved observations are therefore expected to be heavily dominated by $\mathrm{T}$ Tau $\mathrm{S}$ in a ratio of approximately 6:1 (see text for details).

resolution using the Multi-Element Radio-Linked Interferometer Network (MERLIN) and the Very Long Baseline Array (VLBA), which trace the proper motion of the southern radio source and show it to be associated, although not coincident, with T Tau Sb (Smith et al. 2003; Johnston et al. 2004; Loinard et al. 2005, 2007b). These authors show that this small-scale ( $\lesssim 1 \mathrm{au}$ ), compact emission arises from non-thermal gyrosynchrotron radiation, implying a magnetic origin. However, there is another radio component, which is extended (with position angle of $47^{\circ}$ at $8 \mathrm{GHz}$ ), unpolarized, only moderately variable, and has a spectral index typical of optically thin free-free radiation ( $\alpha \simeq 0$; Loinard et al. 2007a). This extended, largescale component is presumed to be diffuse, optically thin emission produced by shocks in the supersonic outflow driven by $\mathrm{T} \mathrm{Tau} \mathrm{Sb}$. Based on these results, we would therefore expect large-scale radio emission from the (unresolved) $\mathrm{T}$ Tau system to be dominated by the shock-ionized plasma of the $\mathrm{T}$ Tau Sb outflow, with only small contributions from the compact, non-thermal component of $\mathrm{T} \mathrm{Tau} \mathrm{Sb}$ and the thermal component from T Tau N.

The recent GMRT observations of $\mathrm{T}$ Tau at 323 and $608 \mathrm{MHz}$ by Ainsworth et al. (2016) did not resolve the separate components of the YSO, but detected an extended source with a 323-608 MHz spectral index of $\alpha_{\mathrm{GMRT}}=$ $0.11 \pm 0.14$, consistent with (partially) optically thin free-free emission. These results are consistent with the extended freefree component of the emission from the $\mathrm{T} \mathrm{Tau} \mathrm{Sb}$ outflow (Loinard et al. 2007a), which is expected to dominate on large scales (several hundred au). Based on their modeling from $323 \mathrm{MHz}$ to $\sim 1 \mathrm{THz}$, Ainsworth et al. (2016) estimated that the frequency of the turnover in the free-free spectrum of this emission is $\sim 200 \mathrm{MHz}$, suggesting that LOFAR observations 
may help constrain the turnover frequency and greatly improve spectral modeling.

In this paper we present the first detection of a YSO with LOFAR at $149 \mathrm{MHz}$. In Section 2 we present details of the observations and data reduction, as well as a discussion of the accuracy of the recovered LOFAR fluxes. In Section 3 we present the resulting radio image of the $\mathrm{T}$ Tau system made at $149 \mathrm{MHz}(2 \mathrm{~m})$ with a bandwidth of $49 \mathrm{MHz}$, along with a measurement of the integrated flux density. In Section 4 we model the spectral energy distribution (SED) and perform an analysis of the fitting parameters. As a result, we place limits on the average electron density, the mass of ionized gas, and the emission measure, using a fitted turnover frequency of $157 \pm 27 \mathrm{MHz}$. We make our concluding remarks in Section 5 .

\section{OBSERVATIONS AND DATA REDUCTION}

The T Tau system was observed for $8 \mathrm{hr}$ with LOFAR (van Haarlem et al. 2013) in high band (HBA) mode over a single night on 2014 January 9-10 (Project code LC1_001). 3C147 was observed as a flux calibrator at the beginning and end of the run. A single beam was used to observe the target field between 110 and $190 \mathrm{MHz}$ using 488 sub-bands with $1 \mathrm{~s}$ integration and 64 channels per sub-band. The data were processed by the default preprocessing pipeline, which performed flagging using AOFLAGGER (Offringa et al. 2012) and averaged the data down to $5 \mathrm{~s}$ integration and four channels per sub-band. While international stations were included in the observation, only data from the Dutch (core and remote) LOFAR stations are used in this paper. This results in a resolution that can be directly compared with the GMRT results of Ainsworth et al. (2016).

Amplitude and clock calibration were performed using the pre-facet calibration pipeline PREFACTOR (van Weeren et al. 2016). This pipeline used the bright calibrator source 3C147 (observed in two 10 minute scans) to calculate the amplitude gains, as well as to separate the clock and TEC (total electron content) phase delay terms from the overall phase delay. As the second (later) scan was of much better quality than the first, only this scan was used to derive the amplitude and clock solutions to be applied to the target field. Note that the TEC delay solutions were not transferred because they are not applicable to the target field. The diagnostic plots produced by PREFACTOR for the lowest and highest $10 \mathrm{MHz}$ of the bandwidth suggested that they were of poor quality and they were excluded from any further processing.

An initial direction-independent phase calibration was applied to the target field using data from the LOFAR global sky model (van Haarlem et al. 2013), a fusion of the VLA Lowfrequency Sky Survey (VLSS, VLSSr; Cohen et al. 2007; Lane et al. 2012), the Westerbork Northern Sky Survey (Rengelink et al. 1997), and the NRAO VLA Sky Survey (Condon et al. 1998). A sky model at LOFAR frequencies was then developed using the actual data. The field was imaged with CASA (McMullin et al. 2007) at five $2 \mathrm{MHz}$ bands at regular intervals across the most sensitive region of bandwidth (with central frequencies of 127.6, 139.4, 151.1, 162.8, and $172.6 \mathrm{MHz}$ ) and the LOFAR source-finding software, PYBDSM (Mohan \& Rafferty 2015), was used to make a multi-frequency sky model of the field. The resulting sky model was then used to generate a new set of directionindependent phase solutions for the original data set, and the process was iterated until two rounds of direction-independent phase-only self-calibration had been applied to the data set. In all cases the standard corrections for the LOFAR beam were applied immediately before imaging.

Several of the brightest sources with strong artefacts were then subtracted using SAGECAL (Kazemi et al. 2011) with the sky model used in the final direction-independent selfcalibration cycle. To achieve the best subtraction and account for a known issue in direction-dependent calibration where the flux of unmodeled sources can be suppressed, SAGECAL was run in "robust" multi-frequency Message Passing Interface mode (Kazemi \& Yatawatta 2013), minimizing flux suppression and ensuring smooth solutions between neighbouring bands. A bright off-field source, 3C123, was also subtracted. Sources to be subtracted were divided into individual SAGECAL solution clusters with unique amplitude and phase solutions, while a single cluster with a single set of amplitude and phase solutions was used to describe the rest of the field. An integration time of 20 minutes was employed and the solutions were applied to the visibilities of the bright sources before subtracting them. The derived solutions for the rest of the field were not applied to the residual visibilities so as to minimize any effect on the target itself.

Final imaging was performed using CASA over approximately $49 \mathrm{MHz}$ of bandwidth centered at $149.1 \mathrm{MHz}$ (subbands 50-300). Only core and remote stations were used and the UV range was clipped to above $1 \mathrm{k} \lambda$. This increased the effective resolution of the image, while suppressing diffuse emission on large scales relative to the anticipated size of the T Tau system in the GMRT images of Ainsworth et al. (2016). Three Taylor terms were employed to describe the sky brightness and $256 \mathrm{~W}$-projection planes were used to image $2^{\circ}$ of the sky with a cell size of $1^{\prime \prime}$. The data were weighted with a Briggs robustness value of zero.

Note that AWIMAGER (Tasse et al. 2013), a LOFAR imager that can apply further corrections for the LOFAR beam during imaging, was also used to image $\mathrm{T}$ Tau. However, as AWIMAGER does not support multi-frequency synthesis imaging with two or more Taylor terms, this resulted in reduced quality due to the large fractional bandwidth in the image. As the CASA image appeared generally of higher quality and primary beam effects at the phase center tend to be small, the result from CASA's CLEAN task using multifrequency synthesis was deemed to be the most accurate. PYBDSM was used to fit the emission from the target, resulting in the Gaussian parameters reported in Section 3.

CASA was used to measure the standard deviation of the flux in a $144 \operatorname{arcmin}^{2}$ box around the T Tau system, yielding a value of $\sigma_{\text {rms }}=0.2 \mathrm{mJy} \mathrm{beam}^{-1}$. Figure 2 shows the spectral indices derived for sources detected in both the $2^{\circ}$ LOFAR image and the $323 \mathrm{MHz}$ GMRT image of Ainsworth et al. (2016). No systematic change in spectral index is evident as sources approach the detection limit of $3 \sigma_{\mathrm{rms}}=0.6 \mathrm{mJy} \mathrm{beam}^{-1}$ of the $149 \mathrm{MHz}$ LOFAR image. This demonstrates that, even without primary beam correction, there is no visible artificial suppression or enhancement of the fluxes of weak sources such as T Tau, at least within the central part of the image.

While Figure 2 shows a stable flux scale across a wide range of fluxes, the absolute accuracy of the local flux scale still needs to be established. Two measures were taken to test this accuracy. First, in order to quantify errors due to elevationdependent gain, the clock and gain solutions were applied to 


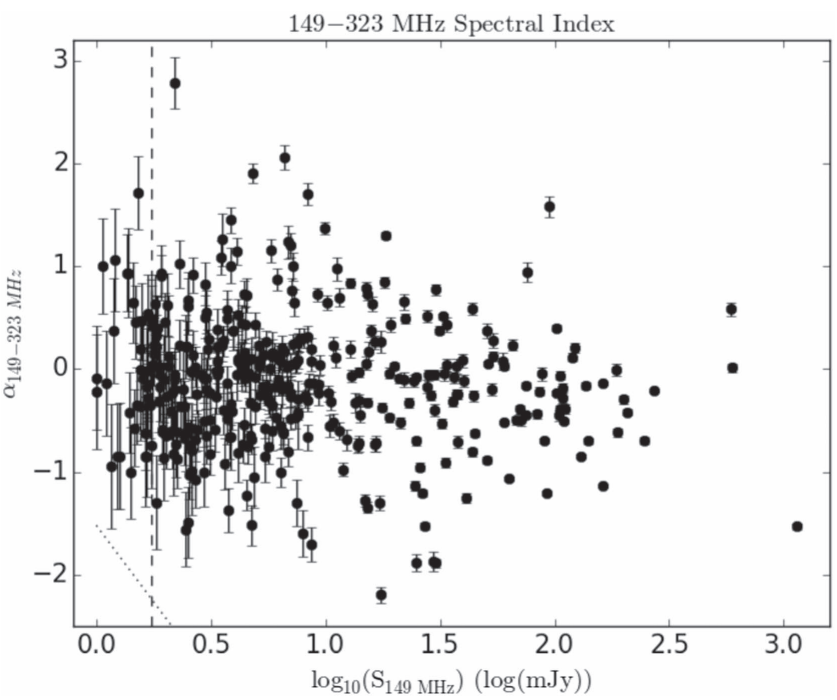

Figure 2. Distribution of spectral index vs. flux density at $149 \mathrm{MHz}$ for all sources detected at $149 \mathrm{MHz}$ with LOFAR and in the $323 \mathrm{MHz}$ GMRT image of Ainsworth et al. (2016). The dashed line indicates the position of T Tau with an integrated flux of $1.75 \pm 0.36 \mathrm{mJy}$. The dotted line shows the $3 \sigma$ sensitivity cutoff for the $323 \mathrm{MHz}$ GMRT image $\left(\sigma_{\mathrm{rms}}=103 \mu \mathrm{Jy}_{\text {beam }}{ }^{-1}\right)$. Error bars indicate $1 \sigma$ non-systematic errors propagated from fits by PYBDSM. There is no evidence for any systematic variation of spectral index with flux, suggesting that significant faint sources in the LOFAR image are free of systematic errors relative to the brighter sources.

the previously unused first scan of $3 \mathrm{C} 147$ (taken $8 \mathrm{hr}$ before the second calibrator scan). Phase-only self-calibration and LOFAR beam corrections were applied in the same way as for the T Tau field. 3C147 was imaged using CASA, recovering a flux of $58.8 \mathrm{Jy}$ at $149.1 \mathrm{MHz}$ compared to the calibrator model flux of $66.7 \mathrm{Jy}$ at $150 \mathrm{MHz}$, which is estimated to be accurate to within $4 \%$ of the true flux (Scaife \& Heald 2012). The mean elevations of the first and second $3 \mathrm{C} 147$ scans were $\sim 40^{\circ}$ and $\sim 64^{\circ}$, respectively, compared with T Tau's mean elevation of $\sim 45^{\circ}$. This implies that the elevation-dependent gain error on the target field is likely less than the $12 \%$ observed between the two $3 \mathrm{C} 147$ scans. No correction was applied to the target field based on this analysis, but the factor of $12 \%$ was considered to be contributing to the systematic uncertainty (see below).

Second, in order to quantify imperfect modeling of LOFAR beam variations across direction and frequency, the integrated LOFAR fluxes of sources within $1^{\circ}$ of T Tau at $149 \mathrm{MHz}$ were compared to the GMRT $150 \mathrm{MHz}$ TGSSADR survey, which has an estimated flux-scale uncertainty of 10\% (Intema et al. 2016) and which also uses the flux scale of Scaife \& Heald (2012). 15 matches were detected within 0.5 of $\mathrm{T}$ Tau, with LOFAR overestimating the GMRT flux by an average of $2 \% .53$ matches were detected within $1^{\circ}$ (inclusive of the 15 within 0.5 ), with LOFAR underestimating the GMRT flux by an average of $6 \%$. Given this good agreement with TGSSADR $150 \mathrm{MHz}$ fluxes, the LOFAR flux scale is likely accurate to within about $10 \%$. We thus conservatively estimate the LOFAR flux scale to be accurate to $12 \%$ in agreement with the 3C147-based testing and have added this figure in quadrature to the Gaussian fit uncertainty, $\sigma_{\text {fit }}$, to calculate the final uncertainty in the integrated flux: $\sigma_{S_{\nu}}=\sqrt{\sigma_{\text {fit }}^{2}+\left(0.12 \times S_{\nu \text {,int }}\right)^{2}}$. Note that $\sigma_{\text {fit }}$ is derived from both the quality of the Gaussian fit and the local rms noise as determined by PYBDSM.

\section{RESULTS}

Figure 3 (left) shows the LOFAR detection of the T Tau system with a peak flux of $S_{149 \mathrm{GHz} \text {,peak }}=0.96 \pm 0.20 \mathrm{mJy} \mathrm{beam}^{-1}$ at a significance of $4.8 \sigma_{\mathrm{rms}}$ excluding systematic errors. The integrated flux is $S_{149 \mathrm{GHz}, \text { int }}=1.75 \pm 0.36 \mathrm{mJy}$ (including the systematic contribution discussed in Section 2) with a Gaussian fit of $(9$ !. $29 \pm 2$ ". 32$) \times(5$.! $80 \pm 1$." 04$)$ and a position angle of $46^{\circ} .8 \pm 23.7$. When compared to the restoring beam of 6 ". $01 \times 4$ ". 90 , this indicates that the emission has been partially resolved at least along one direction. The deconvolved source size was 7 ". $60 \times 1$ !. $^{\prime} 41$ with a position angle of $40^{\circ} .5$. Figure 3 (right) shows the LOFAR data in color with the contours overlaid from the GMRT observations of Ainsworth et al. (2016) at $608 \mathrm{MHz}$. The radio emission appears extended along the same direction at both frequencies and is consistent with the position angle of $47^{\circ}$ of the extended component observed by Loinard et al. (2007a) at $8 \mathrm{GHz}$. As the T Tau system is located at a distance of $148 \mathrm{pc}$ (Loinard et al. 2007b), LOFAR's resolution of $\simeq 5$ !' 4 means that the T Tau system is being probed on a scale of several hundred au. At this resolution, we expect the radio emission to be heavily dominated by $\mathrm{T}$ Tau $\mathrm{S}$ (specifically the extended, free-free component from $\mathrm{T} \mathrm{Tau} \mathrm{Sb)} \mathrm{based} \mathrm{on} \mathrm{the} \mathrm{results} \mathrm{of} \mathrm{Johnston}$ et al. (2003) and Loinard et al. (2007a, see Section 1 and Figure 1), with which indeed the LOFAR detection appears to be consistent.

\section{DISCUSSION}

\subsection{SED Analysis}

The SED for the T Tau system is presented in Figure 4, which combines the flux density measured with LOFAR in this work and the data used in Ainsworth et al. (2016). These authors ensured that integrated fluxes in the free-free, lowfrequency part of the spectrum $(\nu \lesssim 15 \mathrm{GHz})$ were taken from observations with a similar angular resolution so that only emission on similar spatial scales is modeled. LOFAR and the GMRT do not resolve T Tau N and S; therefore where higherresolution VLA data are used (e.g., Skinner \& Brown 1994; Johnston et al. 2003; Loinard et al. 2007a), the T Tau N and S components have been added together and as a result may still have some missing flux. This was also done, in so far as was possible, for the high-frequency $(\nu \gtrsim 15 \mathrm{GHz}$ ) data.

There are two distinct regimes in the SED that need to be modeled: free-free emission associated with the partially ionized outflows at low frequencies and thermal dust emission associated with the circumstellar disks at high frequencies (see discussion in Ainsworth et al. 2016). Although we do not expect the higher-frequency data to contaminate the flux densities at very low frequencies, the long-wavelength tail of the thermal dust emission has been shown to contribute to the centimeter-wave flux densities of YSOs, which can affect the spectral modeling of the entire free-free spectrum and must therefore be included (Scaife et al. 2012).

As the turnover in the free-free spectrum had not yet been detected at GMRT frequencies, Ainsworth et al. (2016) modeled the partially optically thin/thick free-free emission using a single power law with $\alpha=0.17 \pm 0.01$. However, the flux density measured at $149 \mathrm{MHz}$ with LOFAR lies significantly $(3 \sigma)$ below this power law (see Figure 4). Assuming that this drop in flux is due to the transition between optically thin and thick behavior in the free-free spectrum, we now have the spectral constraint necessary to begin to model 


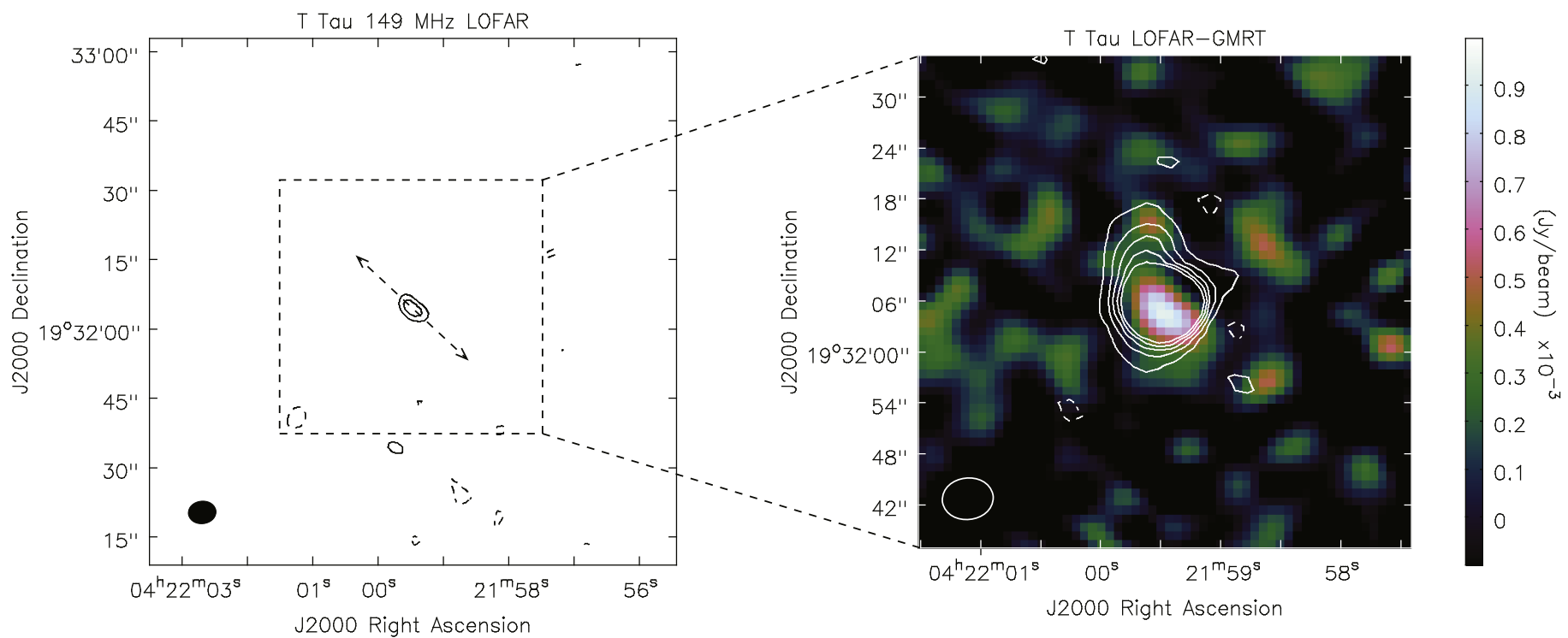

Figure 3. Left: the LOFAR image of the T Tau system at $149 \mathrm{MHz}$ with a CLEAN beam of 6." $01 \times 44^{\prime \prime} 90$ and position angle $276^{\circ} .16$ shown by the filled ellipse. Contours are shown at -3 (dashed), 3 , and $4 \sigma_{\mathrm{rms}}$, where $\sigma_{\mathrm{rms}}$ corresponds to $0.20 \mathrm{mJy}_{\text {beam }}{ }^{-1}$. The dashed line with arrows indicates the axis of the extended component of $\mathrm{T}$ Tau Sb seen at higher frequencies (with position angle of $47^{\circ}$, see Loinard et al. 2007a) with which the LOFAR detection is consistent. The emission has a peak flux of $0.95 \mathrm{mJy}_{\text {beam }}^{-1}$ and an integrated flux of $1.75 \pm 0.36 \mathrm{mJy}$. Right: the color scale shows the $149 \mathrm{MHz}$ LOFAR data. Contours are the GMRT data of Ainsworth et al. (2016) at $608 \mathrm{MHz}$, shown at -3 (dashed), 3, 6, 9, 12, and $15 \sigma_{\mathrm{rms}}$ where $\sigma_{\mathrm{rms}}$ corresponds to $45 \mu \mathrm{Jy}$ beam ${ }^{-1}$. The beam size of the $608 \mathrm{MHz}$ GMRT image is $6{ }^{\prime \prime} 03 \times 5{ }^{\prime \prime} 01$ with position angle $276^{\circ} .18$, which is only $3 \%$ greater than the LOFAR beam area.

the full free-free spectrum. The radio flux density from such emission is given by

$$
\begin{aligned}
\left(\frac{S_{\nu}}{\mathrm{mJy}}\right)= & 7.21586 \times 10^{-4}\left(\frac{\nu}{\mathrm{GHz}}\right)^{2} \\
& \times\left(\frac{T_{\mathrm{e}}}{\mathrm{K}}\right)\left(1-e^{-\tau_{\nu}}\right)\left(\frac{\Omega_{s}}{\operatorname{arcsec}^{2}}\right)
\end{aligned}
$$

(see, e.g., Scaife 2013), where $\tau_{\nu}$ is the optical path length (depth) for free-free emission and can be approximated by

$$
\tau_{\nu}=8.235 \times 10^{-2}\left(\frac{T_{\mathrm{e}}}{\mathrm{K}}\right)^{-1.35}\left(\frac{\nu}{\mathrm{GHz}}\right)^{-2.1}\left(\frac{\mathrm{EM}}{\mathrm{pc} \mathrm{cm}^{-6}}\right)
$$

(Altenhoff et al. 1960). This approximation has an estimated accuracy of over $94 \%$ for LOFAR frequencies and for temperatures of up to $1.5 \times 10^{4} \mathrm{~K}$. In these equations, $T_{\mathrm{e}}$ is the electron temperature and $\Omega_{\mathrm{s}}=\frac{\pi \theta^{2}}{4 \ln 2}$ corresponds to the solid angle of the emission, where $\theta$ is the geometric mean of the deconvolved angular size. EM is the characteristic property of emission measure, which is a function of the average electron number density $\left(n_{\mathrm{e}}\right)$ and source size (Mezger \& Henderson 1967),

$$
\left(\frac{\mathrm{EM}}{\mathrm{pc} \mathrm{cm}^{-6}}\right)=7.1 \times 10^{-3}\left(\frac{D}{\mathrm{kpc}}\right)\left(\frac{\theta}{\operatorname{arcsec}}\right)\left(\frac{n_{\mathrm{e}}}{\mathrm{cm}^{-3}}\right)^{2}
$$

where $D$ is the distance to the source.

We model the flux distribution over the entire range of frequencies using a combination of Equation (1) to model the free-free emission at low frequencies and a power law to model the thermal dust emission at high frequencies. This model has the form

$$
\begin{aligned}
\left(\frac{S_{\nu}}{\mathrm{mJy}}\right)= & 7.21586 \times 10^{-4} \\
& \times\left(\frac{\nu}{\mathrm{GHz}}\right)^{2}\left(\frac{T_{\mathrm{e}}}{\mathrm{K}}\right)\left(1-e^{-\tau_{\nu}}\right)\left(\frac{\Omega_{s}}{\operatorname{arcsec}^{2}}\right) \\
& +K_{100 \mathrm{GHz}}\left(\frac{\nu}{100 \mathrm{GHz}}\right)^{\alpha_{\text {dust }}}
\end{aligned}
$$

where $\alpha_{\text {dust }}$ is the power-law index to be fitted to the highfrequency data and $K_{100 \mathrm{GHz}}$ is a normalization constant that normalizes the high-frequency component of the model at $100 \mathrm{GHz}$.

In a fully optically thick medium $\left(\tau_{\nu} \gg 1\right)$ the observed flux density scales with frequency as $S_{\nu} \propto \nu^{2}$, while in an optically thin medium $\left(1-e^{\tau_{\nu}} \approx \tau_{\nu}\right) S_{\nu} \propto \nu^{-0.1}$ (see, e.g., Scaife 2013). However, in the case of the T Tau system, where a combination of emission from three distinct outflow sources is detected in a medium that may be partially optically thick, or may have contributions to the total flux from both thermal and non-thermal emission, it might be expected that the overall dependence of flux density on frequency is more complicated.

As discussed in Section 1, T Tau S has been shown to dominate the total radio flux density and spectral index over the entire system. However, the steeper $(\alpha \simeq 1)$ thermal emission from $\mathrm{T}$ Tau $\mathrm{N}$ will add a small contribution to the total spectral index of the unresolved $\mathrm{T}$ Tau $\mathrm{N}+\mathrm{S}$ system. Using a leastsquares fitting method in Python, the spectral index of the combined $\mathrm{T}$ Tau $\mathrm{N}+\mathrm{S}$ system is expected to be of order $\alpha \simeq 0.12$ based on the VLA observations of Johnston et al. (2003, see the Appendix). We therefore replace the $\tau_{\nu} \propto \nu^{-2.1}$ dependence (Equation (2)) with $\tau_{\nu} \propto \nu^{\eta}$ in our model to account for this small contribution from $\mathrm{T}$ Tau N. This will also allow for intermediate values of the optical depth from regions where the electron density distribution is different to 

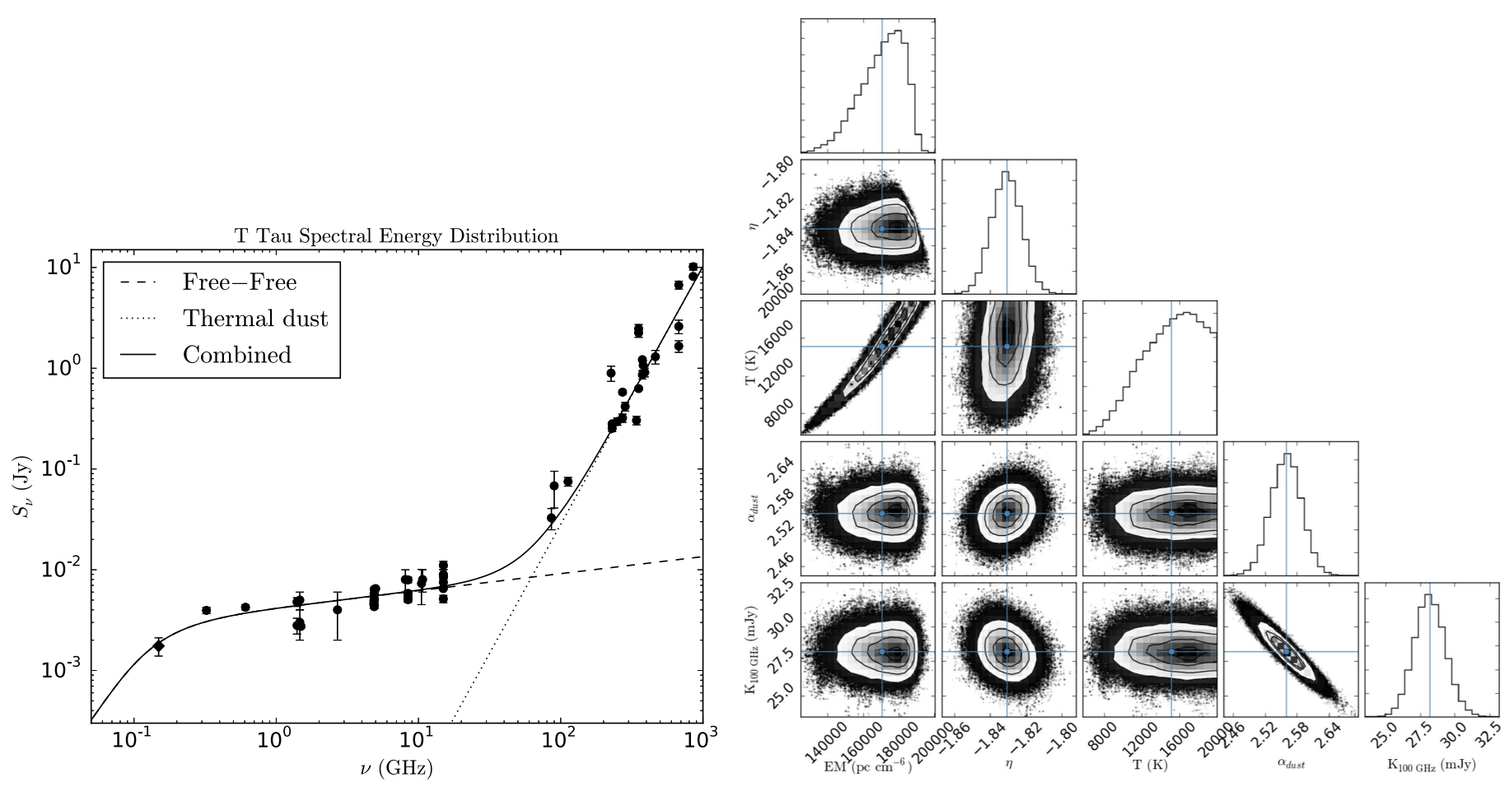

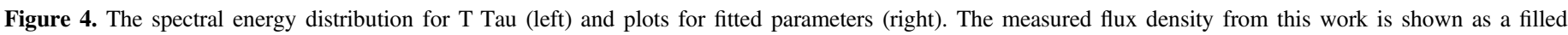

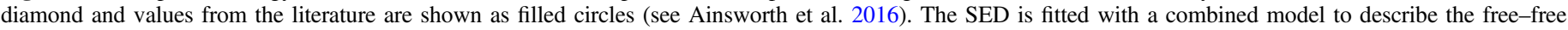

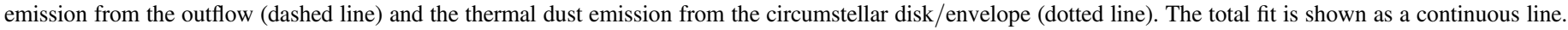

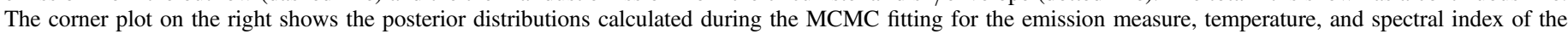

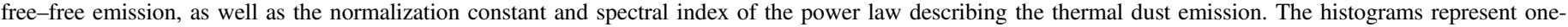

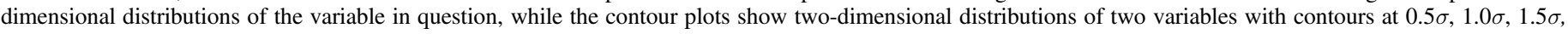
and $2.0 \sigma$. The lines indicate the position of the mean values (see Section 4).

$n_{\mathrm{e}}(r) \propto r^{-2}$ (i.e., where it exhibits a more jet-like geometry, see, e.g., Reynolds 1986) and/or has contributions from nonthermal, gyrosynchrotron emission due to magnetic activity. We note, however, that the gyrosynchrotron radiation has been shown to dominate only the emission detected on small ( $\ll 1 \mathrm{au}$ ) scales (Smith et al. 2003; Loinard et al. 2007a, 2007b), and we expect the total radio emission on large $(\sim 100 \mathrm{au})$ scales to be predominantly associated with free-free radiation due to shock ionization from the outflow driven by $\mathrm{T} \mathrm{Tau} \mathrm{Sb}$ (Loinard et al. 2007a).

The Python Monte Carlo analysis package, PYMC (Patil et al. 2010), was used to find the best fit between Equation (4) and the data in Figure 4 using a Markov chain Monte Carlo (MCMC) approach. The prior ranges used for fitting were $-2.1 \leqslant \eta \leqslant 0,3 \times 10^{3} \leqslant \frac{T_{\mathrm{e}}}{\mathrm{K}} \leqslant 2 \times 10^{4}, 10^{4} \leqslant \frac{\mathrm{EM}}{\mathrm{pc} \mathrm{cm}^{-6}} \leqslant 10^{7}$, $10 \leqslant \frac{K_{100 \mathrm{GHz}}}{\mathrm{mJy}} \leqslant 40$, and $0 \leqslant \alpha_{\text {dust }} \leqslant 4$. The fitting also allowed the posterior distributions of the fitted parameters to be determined, as well the uncertainties in their mean values (see Figure 4).

\subsection{Constraints on Physical Parameters}

Through a measurement of the optically thick surface due to the detection of the turnover frequency, we can break the degeneracy between source size and electron density inherent within the emission measure and constrain the mass of the ionized gas surrounding the T Tau system on scales of several hundreds of au. Figure 4 shows the fitted value of $\mathrm{EM}=(1.67 \pm 0.14) \times 10^{5} \mathrm{pc} \mathrm{cm}^{-6}$ to be well constrained by our SED modeling and we measure a geometric mean of the deconvolved angular source size of $\theta=3$.'27 from our LOFAR image. We then use Equation (3) to find an average electron number density of $n_{\mathrm{e}}=(7.2 \pm 2.1) \times 10^{3} \mathrm{~cm}^{-3}$, and an approximate mass may be derived from this result. Assuming a simple spherical geometry, the density distribution can be integrated to give the ionized gas mass as $M_{\text {ion }}=\frac{4}{3} \pi r_{\text {gas }}^{3}$ $m_{\mathrm{H}} n_{\mathrm{e}}=(1.0 \pm 1.8) \times 10^{-6} M_{\odot}$, where the radius of the sphere $r_{\text {gas }}=D \frac{\theta}{2}$ and $m_{\mathrm{H}}$ is the atomic mass of hydrogen.

The model fitted returns an electron temperature of $T_{\mathrm{e}}=(1.4 \pm 0.3) \times 10^{4} \mathrm{~K}$ for the low-frequency component, a value consistent with the temperature of $10^{4} \mathrm{~K}$ used in the modeling of Ainsworth et al. (2016). It is clear from Figure 4 that this estimate does not constrain the temperature very well because a large range of temperatures from about $10^{4} \mathrm{~K}$ to $1.8 \times 10^{4} \mathrm{~K}$ fit the data almost equally well; thus the fitted value can be regarded only as a rough estimate. Figure 5 shows how the electron density and total ionized mass vary with deconvolved source size and temperature according to Equations (2) and (3) for a spherical gas cloud of constant density. The shaded region indicates the $1 \sigma$ boundary around the measured deconvolved source size and fitted electron temperature reported above.

The high-frequency component was fitted with values of $K_{100 \mathrm{GHz}}=28.3 \pm 1.1 \mathrm{mJy}$ and $\alpha_{\text {dust }}=2.56 \pm 0.03$ (corresponding to a dust opacity index $\beta \approx \alpha_{\text {dust }}-2=0.56$ ). The posterior distributions plotted in Figure 4 show these results to be very well constrained and, as expected, closely matched with the original high-frequency results of Ainsworth et al. (2016). 


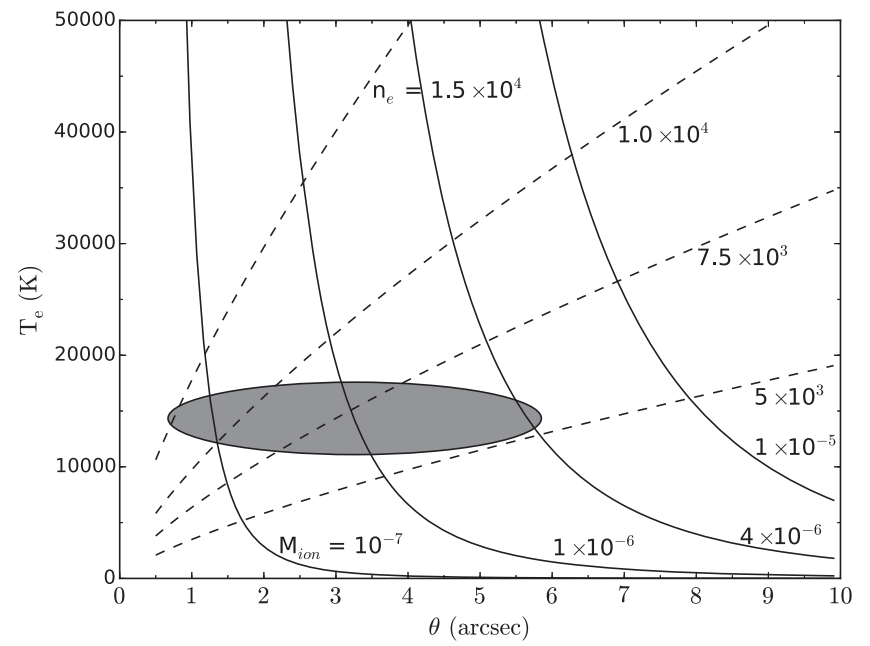

Figure 5. Selected values of electron density $\left(n_{\mathrm{e}}\right.$ in $\left.\mathrm{cm}^{-3}\right)$ and total ionized mass ( $M_{\text {ion }}$ in solar masses) plotted against electron temperature $\left(T_{\mathrm{e}}\right)$ and source size $(\theta)$ using Equations (2) and (3) and assuming a spherical cloud of constant density. The shaded region indicates the area within $1 \sigma$ of the geometric mean of the deconvolved angular source size and fitted temperature $\left(\theta=3\right.$.' $27 \pm 2$ ". $\left.59, T_{\mathrm{e}}=(1.4 \pm 0.3) \times 10^{4} \mathrm{~K}\right)$.

A turnover frequency of $157 \pm 27 \mathrm{MHz}$ was calculated from Equation (2) using the model parameters and setting $\tau_{\nu}=1$. This value is consistent with the interpretation that the lowfrequency turnover of the free-free spectrum has been observed with LOFAR. A mean value of $-1.83 \pm 0.01$ was determined for $\eta$, the modified spectral index term in Equation (2). Thus the flux density scales with $\nu^{0.17}$ above the turnover frequency of $157 \mathrm{MHz}$. This is consistent with the spectral index found in Ainsworth et al. (2016) and does not differ greatly from the canonical relationship of $\nu^{-0.1}$ for optically thin emission, but takes into account the partially optically thick nature of the plasma and the contribution from $\mathrm{T}$ Tau N (see the Appendix).

The spectral index will also be affected by the variability of $\mathrm{T}$ Tau S, which can be clearly seen in Figures 1 and 4 by the range of flux densities measured at different epochs for a given frequency. It is possible that the significant decrease in flux density at $149 \mathrm{MHz}$ is due to variability, as the flux density has been shown to change by up to $50 \%$ in the past (see, e.g., Johnston et al. 2003; Loinard et al. 2007a) and an increase in the flux by up to $50 \%$ would be consistent with the $S_{\nu} \propto \nu^{0.17}$ spectrum.

The variability has been suggested to be due to the compact, non-thermal component associated with $\mathrm{T} \mathrm{Tau} \mathrm{Sb}$ and a range of emission mechanisms have been proposed to produce it, the most likely of which being non-thermal gyrosynchrotron emission (Skinner \& Brown 1994; Johnston et al. 2003; Smith et al. 2003; Loinard et al. 2007a). The spectral index for this component was found to be $\alpha \simeq 0.5$ by Loinard et al. (2007a), which suggests that the peak of the gyrosynchroton spectrum is at higher frequencies and therefore we might expect to see less variability at lower frequencies. This is supported by the multiepoch, multi-frequency observations of Johnston et al. (2003), which show that the flux density of $\mathrm{T}$ Tau $\mathrm{S}$ increases by a factor of approximately four between 1986 and 1988 at $15 \mathrm{GHz}$ and by only a factor of approximately two at $5 \mathrm{GHz}$ (see Figure 1). However, the spectral index of this component has been shown to be variable and helicity reversals of the circular polarization have been observed (see, e.g., Skinner \& Brown 1994; Smith et al. 2003). It is therefore difficult to quantify the relative contribution of this component to the total flux density and variability at LOFAR frequencies.

In the absence of multi-epoch data at these very low frequencies, the LOFAR data suggest a turnover in the freefree spectrum. Simultaneous multi-frequency data extending from LOFAR to VLA frequencies can be used to probe the variability in this regime and constrain these physical properties further.

\section{CONCLUSIONS}

The T Tau system has been successfully detected at $149 \mathrm{MHz}$ with LOFAR. We suggest that this emission is dominated by the extended, free-free component proposed to arise from shock ionization in the outflow of T Tau Sb. This is the lowest-frequency observation of a YSO to date and the first detection of a YSO with LOFAR. The integrated flux of $1.75 \pm 0.36 \mathrm{mJy}$ lies significantly below the power law extrapolated from Ainsworth et al. (2016). Additional LOFAR observations are required to probe the variability of the flux density at $149 \mathrm{MHz}$. In the absence of such multi-epoch observations, we suggest that the turnover in the free-free spectrum has been observed. This flux measurement, along with an estimate of the size of the emitting region based on the partially resolved detection, has allowed the degeneracy between emission measure and electron density to be broken by fitting the SED between $149 \mathrm{MHz}$ and $900 \mathrm{GHz}$. New estimates of the emission measure, electron density, and ionized gas mass have been made. This result establishes the utility of LOFAR as a probe of the spectra of YSOs close to their free-free turnover points; however, sensitivity constraints mean that, for now, only the brightest YSOs will make suitable targets for observation at these low frequencies.

LOFAR, the Low Frequency Array designed and constructed by ASTRON, has facilities in several countries, which are owned by various parties (each with their own funding sources) and which are collectively operated by the International LOFAR Telescope (ILT) foundation under a joint scientific policy. The authors wish to acknowledge the DJEI/DES/SFI/ HEA Irish Centre for High-End Computing (ICHEC) for the provision of computational facilities and support. C.P.C., R.E.A., and T.P.R. would like to acknowledge support from Science Foundation Ireland under grant 13/ERC/I2907. A.M.S. gratefully acknowledges support from the European Research Council under grant ERC-2012-StG-307215 LODESTONE. S.C. acknowledges financial support from the UnivEarthS Labex program of Sorbonne Paris Cité (ANR-10LABX-0023 and ANR-11-IDEX-0005-02).

Software: AOFlagger (Offringa et al. 2012), AWImager (Tasse et al. 2013), CASA (McMullin et al. 2007), Prefactor (van Weeren et al. 2016), PyBDSM (Mohan \& Rafferty 2015), PyMC (Patil et al. 2010), SAGECal (Kazemi et al. 2011; Kazemi \& Yatawatta 2013).

\section{APPENDIX \\ PREDICTED SPECTRAL BEHAVIOR OF THE T TAU N + S SYSTEM}

To ensure that only emission on similar spatial scales is modeled, the SED presented in Figure 4 comprises integrated fluxes taken from observations with similar angular resolutions to these LOFAR observations. However, the LOFAR data 


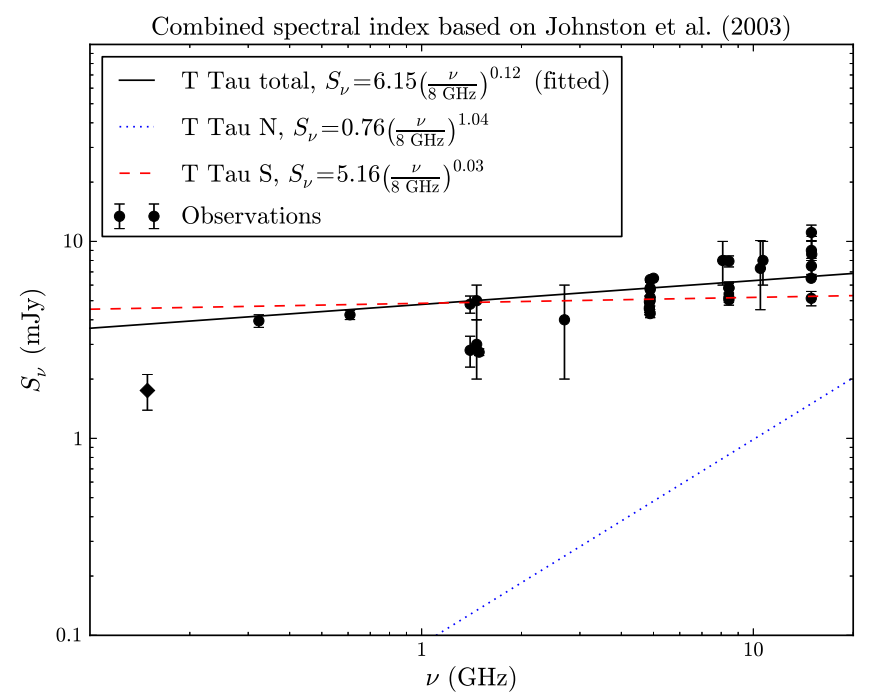

Figure 6. The predicted power-law spectrum for the unresolved $\mathrm{T}$ Tau $\mathrm{N}+\mathrm{S}$ system based on the data presented in Johnston et al. (2003). The (blue) dotted line is the T Tau N spectrum where $0.76 \mathrm{mJy}$ is the time-averaged flux density at $8 \mathrm{GHz}$ and 1.04 is the time-averaged spectral index. The (red) dashed line is the T Tau S spectrum where $5.16 \mathrm{mJy}$ is the time-averaged flux density at $8 \mathrm{GHz}$ and 0.03 is the time-averaged spectral index. The (black) solid line is the fit to the sum of the two power laws using least-squares minimization in Python with normalized flux density of $6.15 \mathrm{mJy}$ at $8 \mathrm{GHz}$ and a spectral index of 0.12 , representative of the $\mathrm{T}$ Tau $\mathrm{N}+\mathrm{S}$ system. Archival observations are shown as filled circles and the LOFAR measurement is a filled diamond, which is clearly discrepant from the fitted spectrum.

presented cannot resolve the $\mathrm{T}$ Tau $\mathrm{N}$ and $\mathrm{S}$ sources and therefore the SED will have contributions from each.

To estimate the overall spectral index above the turnover frequency for the unresolved $\mathrm{T}$ Tau $\mathrm{N}+\mathrm{S}$ system, we fit the sum of two power laws based on the time-averaged data presented in Johnston et al. (2003, see Figure 1) using leastsquares minimization in Python. The power laws are of the form

$$
S_{\nu}=A_{\mathrm{J}, \mathrm{avg}}\left(\frac{\nu}{8 \mathrm{GHz}}\right)^{\alpha_{\mathrm{J}, \mathrm{avg}}} .
$$

$A_{\mathrm{J}, \text { avg }}$ is the $8 \mathrm{GHz}$ flux density averaged over the five epochs measured in Johnston et al. (2003) and is equal to $0.76 \mathrm{mJy}$ for T Tau N and $5.16 \mathrm{mJy}$ for T Tau S. $\alpha_{\mathrm{J}, \text { avg }}$ is the spectral index calculated between 5 and $15 \mathrm{GHz}$ (except for epochs 1989 and 2001, which were calculated between 8 and $15 \mathrm{GHz}$ ) and averaged over the seven epochs measured in Johnston et al. (2003). The average values of spectral index are 1.04 for $\mathrm{T}$ Tau $\mathrm{N}$ and 0.03 for $\mathrm{T}$ Tau $\mathrm{S}$. We note that the T Tau S spectrum itself will have contributions from both the compact/ non-thermal and extended/thermal components associated with gyrosynchrotron radiation from the $\mathrm{T}$ Tau $\mathrm{Sb}$ magnetic fields and free-free radiation from shock-ionized plasma of the $\mathrm{T}$ Tau Sb outflow, respectively. However, these components were unresolved by Johnston et al. (2003) and therefore we do not model them separately here.

The two spectra were then added together and fitted with a single power law, resulting in an overall spectrum with $S_{\nu} \propto \nu^{0.12}$, see Figure 6 . This frequency dependence is close to the $\nu^{0.17}$ measured in Section 4.2 and fits the archival flux density measurements (filled circles in Figure 6). The LOFAR datum (filled diamond) is clearly discrepant from this power law and, if not due to variability, suggests that we have detected the turnover in the free-free spectrum.

\section{REFERENCES}

Ainsworth, R. E., Scaife, A. M. M., Green, D. A., Coughlan, C. P., \& Ray, T. P. 2016, MNRAS, 459, 1248

Ainsworth, R. E., Scaife, A. M. M., Ray, T. P., et al. 2014, ApJL, 792, L18

Altenhoff, W., Mezger, P. G., Strassl, H., Wendker, H., \& Westerhout, G. 1960, Veröffentlichungen Universitäts-Sternwarte Bonn, 59, 48

Anglada, G., Rodríguez, L. F., \& Carrasco-Gonzalez, C. 2015, in Proc. Advancing Astrophysics with the Square Kilometre Array (AASKA14), ed. T. L. Bourke et al., 9

Carrasco-González, C., Rodríguez, L. F., Anglada, G., et al. 2010, Sci, 330, 1209

Cohen, A. S., Lane, W. M., Cotton, W. D., et al. 2007, AJ, 134, 1245

Condon, J. J., Cotton, W. D., Greisen, E. W., et al. 1998, AJ, 115, 1693

Dyck, H. M., Simon, T., \& Zuckerman, B. 1982, ApJL, 255, L103

Dzib, S. A., Loinard, L., \& Rodríguez, L. F. 2015, ApJ, 801, 91

Gustafsson, M., Kristensen, L. E., Kasper, M., \& Herbst, T. M. 2010, A\&A, 517, A19

Intema, H. T., Jagannathan, P., Mooley, K. P., \& Frail, D. A. 2016, A\&A, submitted (arXiv:1603.04368)

Johnston, K. J., Fey, A. L., Gaume, R. A., et al. 2004, ApJL, 604, L65

Johnston, K. J., Gaume, R. A., Fey, A. L., de Vegt, C., \& Claussen, M. J. 2003, AJ, 125,858

Joy, A. H. 1945, ApJ, 102, 168

Kazemi, S., \& Yatawatta, S. 2013, MNRAS, 435, 597

Kazemi, S., Yatawatta, S., Zaroubi, S., et al. 2011, MNRAS, 414, 1656

Köhler, R., Ratzka, T., Herbst, T. M., \& Kasper, M. 2008, A\&A, 482, 929

Koresko, C. D. 2000, ApJL, 531, L147

Lane, W. M., Cotton, W. D., Helmboldt, J. F., \& Kassim, N. E. 2012, RaSc, 47, RS0K04

Loinard, L., Mioduszewski, A. J., Rodríguez, L. F., et al. 2005, ApJL, 619, L179

Loinard, L., Rodríguez, L. F., D’Alessio, P., Rodríguez, M. I., \& González, R. F. 2007a, ApJ, 657, 916

Loinard, L., Torres, R. M., Mioduszewski, A. J., et al. 2007b, ApJ, 671, 546

McMullin, J. P., Waters, B., Schiebel, D., Young, W., \& Golap, K. 2007, in ASP Conf. Ser. 376, Astronomical Data Analysis Software and Systems XVI, ed. R. A. Shaw, F. Hill, \& D. J. Bell (San Francisco, CA: ASP), 127

Mezger, P. G., \& Henderson, A. P. 1967, ApJ, 147, 471

Mohan, N., \& Rafferty, D. 2015, ASCL, PyBDSM: Python Blob Detection and Source Measurement, ascl:1502.007

Offringa, A. R., van de Gronde, J. J., \& Roerdink, J. B. T. M. 2012, A\&A, 539, A95

Padovani, M., Marcowith, A., Hennebelle, P., \& Ferrière, K. 2016, A\&A, 590, A8

Patil, A., Huard, D., \& Fonnesbeck, C. J. 2010, J. Stat. Softw., 35, 4

Ray, T. P., Muxlow, T. W. B., Axon, D. J., et al. 1997, Natur, 385, 415

Reipurth, B., Bally, J., \& Devine, D. 1997, AJ, 114, 2708

Rengelink, R. B., Tang, Y., de Bruyn, A. G., et al. 1997, A\&AS, 124, 259

Reynolds, S. P. 1986, ApJ, 304, 713

Scaife, A. M. M. 2011, ATel, 3786

Scaife, A. M. M. 2013, AdAst, 2013, 14

Scaife, A. M. M., Buckle, J. V., Ainsworth, R. E., et al. 2012, MNRAS, 420, 3334

Scaife, A. M. M., \& Heald, G. H. 2012, MNRAS, 423, L30

Schwartz, P. R., Simon, T., \& Campbell, R. 1986, ApJ, 303, 233

Skinner, S. L., \& Brown, A. 1994, AJ, 107, 1461

Smith, K., Pestalozzi, M., Güdel, M., Conway, J., \& Benz, A. O. 2003, A\&A, 406, 957

Tasse, C., van der Tol, S., van Zwieten, J., van Diepen, G., \& Bhatnagar, S. 2013, A\&A, 553, A105

van Haarlem, M. P., Wise, M. W., Gunst, A. W., et al. 2013, A\&A, 556, A2 van Weeren, R. J., Williams, W. L., Hardcastle, M. J., et al. 2016, ApJS, 223, 2 